https://doi.org/10.21516/2072-0076-2020-13-3-21-29

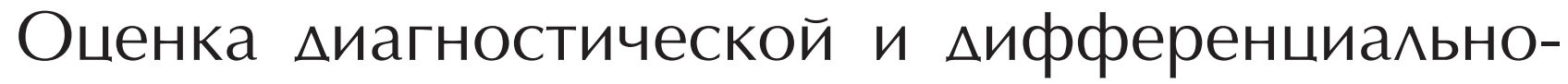 Аиагностической значимости мето $о в$ спектральной оптической когерентной томографии и микропериметрии у пациентов со зрительными нарушениями вследствие рассеянного склероза
}

\author{
М.С. Кривошеева', Е.Э. Иойлева², 3 \\ 1 ГБУЗ Московской области «Сергиево-Посадская районная больница», Новоугличское шоссе, д. 62a, \\ Московская область, г. Сергиев Посад, 141301, Россия \\ 2 ФГАУ НМИЦ «МНТК "Микрохирургия глаза" им. акад. С.Н. Федорова» Минздрава России, Бескудниковский бульвар, \\ д. 59а, Москва, 127486, Россия \\ ${ }^{3}$ МГМСУ им. А.С. Евдокимова, ул. Делегатская, д. 20, стр. 1, Москва, 127473, Россия
}

Цель - статистическая оценка диагностической и дифференциально-диагностической значимости спектральной оптической когерентной томографии (СОКТ) и микропериметрии у пациентов (1) с оптическим невритом (ОН) при манифестации рассеянного склероза (PC), (2) с ОН воспалительной этиологии и (3) атрофией зрительного нерва (АЗН) вследствие РС. Материал и методы. Результаты обследования трех групп пациентов (групп ОН и АЗН вследствие РС и группы ОН воспалительной этиологии) обработаны методами статистического анализа (корреляционного, многомерного и методом бинарной логистической регрессии). Порог электрической чувствительности (ПЭЧ) и электрическую лабильность (ЭЛ) определяли на приборе «Фосфен-тестер» по стандартной методике. СОКТ проводили на Cirrus HD-OCT(Carl Zeiss Meditec Inc., USA) nо программам RNFL Thickness Analysis, Ganglion Cell Analysis, компьютерную микропериметрию - на MP-1 (Nidek technologies, Vigonza, Italy) с применением программы Масиа $12^{\circ} 10$ дБ. Результаты. Выявлена корреляционная связь ПЭЧ сетчатки с показателем средней светочувствительности сетчатки, средней толщиной перипапиллярного слоя нервных волокон сетчатки (СНВС), объемом верхневисочного и верхнего секторов комплекса ганглиозных клеток сетчатки с внутренним плексиформным слоем (ГКС + ВПС). Выявлены и подтверждены значимые при поражении зрительного анализатора вследствие РС диагностические параметры снижение средней светочувствительности сетчатки в пределах до $6^{\circ}$ от центра поля зрения по данным микропериметрии $(p<0,0005)$, снижение толщины СНВС $(p<0,005)$ и комплекса ГКС + ВПС по данным СОКТ $(p=0,004)$. При проведении внутригрупповых сравнений выявлена диагностическая значимость СОКТ в ранней диагностике АЗН вследствие РС (p <0,0005). Выявлено, что если показатель СНВС составляет 62 мкм и менее, а ГКС + ВПС - 52 мкм и менее (в нижнем сегменте 50 мкм и менее, а в верхнем сегменте - 51 мкм и менее), то это с чувствительностью $100 \%$ и специфичностью 87,5-93 \% свидетельствует о наличии АЗН. Заключение. Средняя светочувствительность сетчатки в пределах до $6^{\circ}$ от центра поля зрения, толщина СНВС, ГКС + ВПС, в том числе в верхнем, верхневисочном и нижнем сегментах, являются диагностически значимыми показателями при ОН воспалительной и демиелинизирующей этиологии и развитии АЗН вследствие РС.

Ключевые слова: рассеянный склероз; оптический неврит; атрофия зрительного нерва; спектральная оптическая когерентная томография; микропериметрия; корреляционный анализ; многомерный анализ; регрессионный анализ Конфликт интересов: отсутствует.

Прозрачность финансовой деятельности: никто из авторов не имеет финансовой заинтересованности в представленных материалах или методах.

Для цитирования: Кривошеева М.С., Иойлева Е.Э. Оценка диагностической и дифференциально-диагностической значимости методов спектральной оптической когерентной томографии и микропериметрии у пациентов со зрительными нарушениями вследствие рассеянного склероза. Российский офтальмологический журнал. 2020 ; 13 (3): $21-29$.

https://doi.org/10.21516/2072-0076-2020-13-3-21-29 


\title{
Evaluation of the diagnostic and differential diagnostic value of SOCT and microperimetry methods in patients with visual disturbances due to multiple sclerosis
}

\author{
Maria S. Krivosheeva', Elena E. loyleva ${ }^{2,3}$ \\ ${ }^{1}$ City hospital of Sergiev Posad, Novouglichskoe highway, 62a, Sergiev Posad, Moscow region, 141301, Russia \\ 2 S.N. Fedorov "Eye Microsurgery NMRC", 59a, Beskudnikovsky Boulevard, Moscow, 127486, Russia \\ ${ }^{3}$ A.I. Evdokimov Moscow State University of Medicine and Dentistry, 20, Bldg.1, Delegatskaja St., Moscow, 127473, Russia \\ krivosheeva_ms@mail.ru
}

Purpose: statistical evaluation of the diagnostic and differential diagnostic significance of spectral optical coherent tomography (SOCT) and microperimetry methods in patients with (1) optic neuritis (ON) with multiple sclerosis (MS) manifestation; (2) inflammation-related ON and (3) optic nerve atrophy (ONA) caused by MS. Materials and methods. The results of examination of the three groups of patients were processed by correlation and multivariate analysis and binary logistic regression. The threshold of electrical sensitivity (EST) and electrical lability (EL) were determined on a Fosphen-tester using a standard technique. S-OCT was performed on Cirrus HD-OCT (Carl Zeiss Meditec Inc., USA) using the RNFL Thickness Analysis, Ganglion Cell Analysis programs. Computer microperimetry was done on MP-1 (Nidek technologies, Vigonza, Italy) using the macula $12^{\circ} 10 \mathrm{~dB}$ program. Results. EST of the retina was found to have a strong bilateral correlation with the average retinal sensitivity, RNFL thickness and thickness of the upper temporal and upper segments layer of the complex $G C L+I P L$. Diagnostic parameters significant for visual analyzer disorders caused by MS were revealed and confirmed, including the average retinal sensitivity drop within $6^{\circ}$ from the center of the visual field according to microperimetry $(p<0.0005)$, the thinning of $R N F L$ ( $\left.p<0.005\right)$ and complex $G C L-S+I P L$, according to SOCT $(p=0.004)$. intragroup comparisons revealed the diagnostic significance of SOCT in early diagnosis of ONA due to MS ( $p<0.0005)$. It was found that if the RNFL was 62 microns or less, the GCL-S + IPL was 52 microns or less (in the inferior segment it was 50 microns or less, and in the upper segment it was 51 microns or less) then, with a sensitivity of $100 \%$ and a specificity of $87.5-93 \%$, it is an evidence of the atrophy of the optic nerve. Conclusion: average retinal photosensitivity within $6^{\circ}$ from the center of the visual field, RNFL thickness, thickness GCL-S +IPL, including that in the superior, superior temporal and inferior segments can be considered as diagnostically significant values in OH inflammatory-related and demyelinating OH and the development of ONA due to MS.

Keywords: multiple sclerosis; optic neuritis, optic nerve atrophy; spectral optical coherence tomography; microperimetry; correlation analysis; multivariate analysis; regression analysis

Conflict of interests: there is no conflict of interests.

Financial disclosure: No author has a financial or property interest in any material or method mentioned.

For citation: Krivosheeva M.S., Ioyleva E.E. Evaluation of the diagnostic and differential diagnostic value of SOCT and microperimetry methods in patients with visual disturbances due to multiple sclerosis. Russian ophthalmological journal. 2020; 13 (3): 21-9 (In Russian). https://doi.org/10.21516/2072-0076-2020-13-3-21-29

Рассеянный склероз (РС) - хроническое заболевание центральной нервной системы (ЦНС). Для него характерно диффузное поражение головного мозга (ГМ) с ранним вовлечением в процесс осевых цилиндров аксонов, приводящее к полиморфизму клинических проявлений заболевания с развитием тяжелого неврологического дефицита [1, 2]. Манифестация заболевания может наблюдаться как у детей, так и у людей среднего возраста, но чаще встречается в молодом возрасте, с течением времени приводит к развитию инвалидности вследствие необратимых неврологических нарушений и снижения зрительных функций [3]. Среди всех причин инвалидности лиц молодого возраста вследствие неврологических заболеваний РС занимает второе ранговое место и составляет $19,5 \%$ от всех случаев [4].

Для диагностики зрительных нарушений при РС применяются методы структурно-топографического (спектральная оптическая когерентная томография, СОКТ) и функционального анализа (компьютерная периметрия, определение порога электрической чувствительности - ПЭЧ и электрической лабильности - ЭЛ). Существующие исследования, посвященные зрительным нарушениям при оптическом неврите $(\mathrm{OH})$, в том числе и демиелинизирующей этиологии, выполнены на немногочисленных выборках пациентов [5, 6]. В связи с этим оценка дифференциально-диагностической значимости методов структурно-топографического анализа на большой выборке пациентов с РС с помощью расширенной обработки данных инструментами статистики высокого порядка является актуальной.

Современные методы статистического анализа, рекомендованные международными научными сообществами как инструмент доказательной медицины, широко используются в научных исследованиях [7, 8]. Наиболее распространенным статистическим методом обработки данных является корреляционный анализ (correlation analysis), суть которого заключается в обнаружении линейной зависимости между двумя случайными признаками или факторами [9]. Зависимость между тремя и большим числом случайных признаков или факторов изучается методом многомерного анализа (multivariate analysis), направленным на выявление характера и структуры взаимосвязей между компонентами, 
что позволяет произвести оценку взаимодействия факторов (параметров) [10]. Регрессионный анализ (regression analysis) заключается в проверке статистической гипотезы значимости связи между величинами, выявленной в результате корреляционного и/или многомерного анализа. Целью регрессионного анализа является определение общего вида уравнения регрессии и проверка статистических гипотез о регрессии [11].

Таким образом, на основании всех данных, полученных нами в процессе многолетнего исследования пациентов с РС и с учетом клинических рекомендаций по статистическому анализу, ЦЕЛЬЮ работы явилась оценка диагностической и дифференциально-диагностической значимости COKT и микропериметрии у пациентов с ОН при манифестации РС, ОН воспалительной этиологии и атрофией зрительного нерва (АЗН) вследствие РС методами корреляционного, многомерного и регрессионного анализа.

\section{МАТЕРИАЛ И МЕТОДЫ}

В исследование включено 156 пациентов, из них 142 пациента с диагнозом РС, подтвержденным врачом-неврологом на основании данных нейровизуализации головного мозга согласно международным критериям Мак Дональда 2010 г. пересмотра и 14 пациентов с клиникой ОН без подтвержденного диагноза РС, составивших группу сравнения (табл. 1). Контроль полученных результатов проводился на основании эталонных значений возрастной нормы, полученных при обследовании 28 соматически здоровых добровольцев с использованием тех же диагностических систем, что и у основных групп пациентов и группы сравнения. Из исследования были исключены лица с аметропиями средней и высокой степени, общей соматической и/или глазной патологией, в том числе в результате полученных травм и/ или интоксикаций, влияющих на результаты обследования. Все лица, участвующие в исследовании, подписали информированное согласие на участие в исследовании в соответствии с нормативными документами (Хельсинкская декларация всемирной медицинской ассоциации «Этические принципы проведения научных медицинских исследований с участием человека» с поправками 2000 г. и «Правила клинической практики в Российской Федерации», утвержденные Приказом Минздрава РФ от 19.06.2003 № 266).

Обследование офтальмологического статуса и подтверждение диагноза ОН и частичной АЗН проводились в ФГАУ НМИЦ «МНТК "Микрохирургия глаза" им. акад. С.Н. Федорова» МЗ РФ. ПЭЧ и ЭЛ оценивали с помощью прибора «Фосфен-тестер» по стандартной методике. Толщину перипапиллярного слоя нервных волокон сетчатки (СНВС) и комплекса ганглиозных клеток сетчатки (ГКС) определяли с помощью СОKT (Cirrus HD-OCT Carl Zeiss Meditec Inc., USA) по протоколам Optic Disc Cube $200 \times 200$ в программе RNFL Thickness Analysis, Ganglion Cell Analysis. Компьютерная микропериметрия (MP-1, Nidek technologies, Vigonza, Italy) проводилась по программе Macula $12^{\circ} 10$ дБ, обследовалась центральная зона в пределах $12^{\circ}$ от центра фиксации путем последовательного предъявления 68 стандартных световых стимулов Goldmann III размером $0,43^{\circ}$.

Обследованы оба глаза всех трех групп пациентов (групп ОН и АЗН вследствие РС и группы ОН воспалительной этиологии) с проведением статистического анализа (корреляционного, многомерного, бинарной логистической регрессии). Острота зрения с коррекцией составила $0,1-0,8$, у всех пациентов характер зрения был бинокулярным. Показатели рефракции и внутриглазного давления (ВГД) у всех пациентов находились в пределах нормальных значений.

В группе с ОН вследствие РС были выделены пораженный (с невритом) и парный глаза (без неврита). Офтальмоскопическая картина пораженных глаз варьировала от проминенции диска зрительного нерва (ДЗН) с выраженным отеком перипапиллярного слоя сетчатки и извитостью вен до минимальных признаков отека и извитости вен.

В группе сравнения - пациенты с ОН воспалительной этиологии - диагноз РС не был подтвержден, но установлена причина неврита - воспалительный процесс ЛОР-органов. Офтальмоскопическая картина пораженных глаз представляла собой выраженную проминенцию ДЗН со множеством патологических рефлексов глазного дна.

В группе пациентов с АЗН вследствие РС, которые были обследованы впервые, диагноз был установлен на основании снижения остроты зрения, истончения СНВС по данным СОКТ и офтальмоскопии. Был выделен худший и лучший в функциональном отношении глаз в зависимости от остроты зрения и средней толщины СНВС. Офтальмоскопическая картина как худшего, так и лучшего глаза варьировала от отсутствия видимых изменений на глазном дне до побледнения височного сегмента.

При статистической обработке использовали результаты микропериметрии: показатель средней светочувствительности сетчатки (ПСС), а также СОКТ: среднюю толщину перипапиллярного CHBC (RNFL Thickness), толщину слоя нервных волокон в височном (RNFL-T), носовом (RNFL-N), верхнем (RNFL-S) и нижнем (RNFL-I) квадрантах, среднюю толщину комплекса ГКС с внутренним плексиформным слоем (ГКС + ВПС) с его сегментарной оценкой. Кроме того, в исследование диагностической значимости были включены показатели ПЭЧ и ЭЛ, поскольку их значения были изменены в каждой клинической группе, а также в группе сравнения и при проведении предварительного анализа была выявлена корреляционная связь с показателями СОКТ и микропериметрии.

Обработка данных осуществлялась с использованием программных пакетов Microsoft Excel 2010 (Microsoft Office),

Таблица 1. Распределение пациентов в основных группах и группе сравнения по возрасту и полу

Table 1. Distribution of patients in the main groups and the comparison group by age and sex

\begin{tabular}{|c|c|c|c|}
\hline \multirow[t]{2}{*}{$\begin{array}{l}\text { Группы } \\
\text { Groups }\end{array}$} & \multirow{2}{*}{$\begin{array}{c}\text { Средний возраст, лет, } \mathrm{M} \pm \sigma \\
\text { (мин-макс) } \\
\text { Average Age, yrs, } \mathrm{M} \pm \sigma \\
\text { (min-max) }\end{array}$} & \multicolumn{2}{|c|}{$\begin{array}{l}\text { Пол } \\
\text { Sех }\end{array}$} \\
\hline & & $\begin{array}{c}\text { мужчины } \\
\text { men }\end{array}$ & $\begin{array}{l}\text { женщины } \\
\text { women }\end{array}$ \\
\hline $\begin{array}{l}\text { 1-я основная: оптический неврит } \\
1^{\text {st }} \text { main group: optic neuritis }\end{array}$ & $26,4 \pm 2,8(21-41)$ & 14 & 48 \\
\hline $\begin{array}{l}\text { 2-я основная: атрофия зрительного нерва } \\
2^{\text {nd }} \text { main group: optic nerve atrophy }\end{array}$ & $35,6 \pm 3,7(24-56)$ & 36 & 44 \\
\hline $\begin{array}{l}\text { 3-я сравнения: оптический неврит воспалительной этиологии } \\
3^{\mathrm{d}} \text { comparison group: optic neuritis of inflammatory etiology }\end{array}$ & $25,7 \pm 1,6(22-37)$ & 2 & 12 \\
\hline
\end{tabular}

Российский офтальмологический журнал, 2020; 13(3): 21-9 
IBM SPSS Statistics 23.0 на базе отделения медицинской статистики. Количественные переменные представляли в виде $\mathrm{M} \pm \sigma$ (среднее значение \pm стандартное отклонение), в отдельных случаях в виде $\mathrm{M} \pm \mathrm{m}$ (ошибка среднего); порядковые и качественные переменные - в виде доли от общего количества по категориям. Во всех случаях использовали двусторонние варианты статистических тестов, нулевую гипотезу отвергали при $\mathrm{p}<0,05$. Нормальность распределения количественных переменных тестировали по критериям Колмогорова - Смирнова и Шапиро - Уилка. В случае нормального распределения переменных в корреляционном анализе использовали коэффициент корреляции Пирсона, в случае несоответствия хотя бы одной переменной нормальному распределению использовали коэффициент корреляции Спирмена.

Для оценки значимости эффекта фактора группы с учетом пораженного/парного глаза на отдельные переменные каждого диагностического теста использовали общую линейную модель с повторными измерениями, апостериорные попарные сравнения проводили с помощью множественных межгрупповых сравнений с поправками Шефе, наименьшей значимой разницы, Бонферрони и двустороннего теста Даннета. Оценка значимости эффекта факторов на результаты каждого диагностического теста проводилась с использованием многомерных критериев, таких как след Пиллаи, лямбда Уилкса, след Хотеллинга и наибольший корень Роя. Оценка различий по каждому диагностическому критерию проводилась с помощью множественных межгрупповых сравнений в виде апостериорных тестов с поправками Шефе, наименьшей значимой разницы, Бонферрони и двустороннего теста Даннета. Для определения влияния фактора группы на результаты коррелирующих параметров каждого диагностического теста использовали многомерный тест ANOVA.

Для выявления дифференциально-диагностической ценности методов структурно-топографического и функционального анализа использовали бинарную логистическую регрессию с последовательным включением в модель значимых параметров по Вальду.

\section{РЕЗУЛЬТАТЫ}

Корреляционный анализ. В предыдущих исследованиях нами было показано повышение показателя ПЭЧ и снижение ЭЛ различной степени у пациентов с ОН и АЗН вследствие PC, а также с ОН воспалительной этиологии [12-14]. В данном исследовании проводился глобальный поиск клиникофункциональных взаимосвязей. Так, при оценке данных трех исследуемых групп пациентов методом корреляционного анализа было выявлено, что на пораженном (худшем в функциональном отношении) глазу показатель ПЭЧ сетчатки имеет сильную двустороннюю корреляционную связь с ПСС в пределах до $6^{\circ}$ от центра поля зрения, подтвержденную как минимум тремя статистическими тестами. Кроме того, ПЭЧ сетчатки имеет корреляционные связи с параметрами СОКТ - средней толщиной СНВС, объемом верхневисочного и верхнего сегментов комплекса ГКС + ВПС, что представлено в таблицах 2 и 3.

Многомерный анализ. Показатель ПЭЧ сетчатки демонстрировал высокий уровень статистически значимых различий между пораженным и парным глазом ( $\mathrm{p}=0,019)$, между клиническими группами в целом уровень статистически значимых различий был низким ( $\mathrm{p}=0,351)$, однако отмечалась разница между глазами пациентов в разных группах.

Таблица 2. Коррелирующие параметры по данным разных методов исследования на пораженном (худшем) глазу

Table 2. Correlated parameters according to different research methods (affected (worst) eye)

\begin{tabular}{|c|c|c|c|}
\hline $\begin{array}{l}\text { Кopрелирующие параметры } \\
\text { Correlated parameters }\end{array}$ & $\begin{array}{c}\text { Коэффициент корреляции } \\
\text { Пирсона } \\
\text { Pearson correlation coefficient }\end{array}$ & $\begin{array}{c}\text { Коэффициент Тау-b } \\
\text { Кендалла } \\
\text { Kendall Tay-b coefficient } \\
\end{array}$ & $\begin{array}{c}\text { Коэффициент корреляции } \\
\text { Спирмена Po } \\
\text { Spearman correlation coefficient Po }\end{array}$ \\
\hline $\begin{array}{l}\text { ПЭЧ - ПСС } \\
\text { Retinal electrical sensitivity - } \\
\text { mean sensitivity }\end{array}$ & $0,422^{*}$ & $0,335^{*}$ & $0,469^{*}$ \\
\hline $\begin{array}{l}\text { ЭЛ - ПСС } \\
\text { Electrical lability }- \text { mean } \\
\text { sensitivity }\end{array}$ & $0,55^{*}$ & $0,347^{*}$ & $0,48^{*}$ \\
\hline $\begin{array}{l}\text { ПЭЧ - CHВС } \\
\text { Retinal electrical sensitivity - } \\
\text { RNFL thickness }\end{array}$ & $0,217^{* *}$ & - & - \\
\hline $\begin{array}{l}\text { ПЭЧ - ГКС + ВПС } \\
\text { Mean sensitivity - GCL + IPL } \\
\text { complex }\end{array}$ & $0,453^{* *}$ & - & - \\
\hline
\end{tabular}

Примечание. * $\mathrm{p}<0,01 ; *$ p $<0,05$.

Note. ${ }^{*} \mathrm{p}<0.01 ;{ }^{* *} \mathrm{p}<0.05$. RNFL - retinal nerve fiber layer, GCL - ganglion cells layer, IPL - intern plexiform layer.

Таблица 3. Коррелирующие параметры по данным разных методов исследования на парном (лучшем) глазу

Table 3. Correlated parameters according to different research methods (fellow (best) eye)

\begin{tabular}{|l|c|c|c|}
\hline $\begin{array}{l}\text { Koppeлирующие параметры } \\
\text { Correlated parameters }\end{array}$ & $\begin{array}{c}\text { Коэффициент корреляции } \\
\text { Пирсона }\end{array}$ & $\begin{array}{c}\text { Коэффициент Тау-b } \\
\text { Кендалла } \\
\text { Kendall Тау-b coefficient }\end{array}$ & $\begin{array}{c}\text { Коэффициент корреляции } \\
\text { Спирмена Ро } \\
\text { Spearman correlation coefficient Po }\end{array}$ \\
\hline $\begin{array}{l}\text { ПЭЧ - ПСС } \\
\text { Retinal electrical sensitivity - } \\
\text { mean sensitivity }\end{array}$ & $0,312^{*}$ & $0,231^{*}$ & $0,38^{*}$ \\
\hline $\begin{array}{l}\text { ПCС - ГКС + ВПС } \\
\text { Mean sensitivity - GCL + IPL } \\
\text { complex }\end{array}$ & $0,354^{*}$ & - & - \\
\hline
\end{tabular}

Примечание. * $\mathrm{p}<0,05$.

Note. ${ }^{*} \mathrm{p}<0.05$, GCL - ganglion cells layer, IPL - intern plexiform layer. 
При ОН воспалительной и демиелинизирующей этиологии были выявлены статистически значимые различия ПЭЧ сетчатки на пораженных глазах $(86,85$ мкА к 65,62 мкА для ОН воспалительной этиологии и 111,26 мкА к 69,48 мкА для ОН вследствие РС соответственно), а в группе АЗН вследствие РС уровень статистической значимости различий по наблюдаемому показателю между пораженным и парным глазами был низкий (79,54 мкА и 86,87 мкА соответственно). Выявлен высокий уровень статистической значимости взаимодействия фактора группы и фактора глаза $(\mathrm{p}=0,001)$. С помощью дополнительных попарных сравнений между группами выявлено, что для пораженного глаза уровень статистической значимости различий по ПЭЧ сетчатки выше при ОН вследствие РС, чем при ОН воспалительной этиологии и АЗН вследствие РС $(\mathrm{p}<0,05)$; для парного глаза уровень статистической значимости по ПЭЧ сетчатки выше при АЗН вследствие РС, чем при ОН воспалительной и демиелинизирующей этиологии $(\mathrm{p}<0,05)$.

Выявлен высокий уровень статистической значимости различий по ПСС сетчатки (по данным микропериметрии) между пораженным и парным глазами для всех групп (показатель для пораженного глаза значимо ниже, $\mathrm{p}<0,0005)$. Межгрупповой уровень статистической значимости различий по ПСС сетчатки был низким $(\mathrm{p}=0,15)$.

С помощью многомерных критериев подтверждена высокая диагностическая значимость параметра толщины СНВС/ RNFL Thickness. Отмечен высокий уровень статистической значимости различий данного параметра в исследуемых группах в целом (109,15 мкм для ОН воспалительной этиологии, 81,1 мкм для АЗН вследствие РС , 97,20 мкм для ОН вследствие $\mathrm{PC}, \mathrm{p}<0,0005$; попарные сравнения между группами также значимы). Паттерн различий между пораженным и парным глазами для разных групп был неодинаков (взаимодействие факторов группы и глаза статистически значимо, $\mathrm{p}=0,001$, табл. 4).

В группе ОН воспалительной и демиелинизирующей этиологии толщина СНВС оказалась больше на пораженном глазу, чем на парном (121,39 и 96,92 мкм для ОН воспалительной этиологии, 105,81 и 88,58 мкм для ОН вследствие РС соответственно). В группе АЗН вследствие РС уровень статистической значимости различий был низким (78,76 и 83,44 мкм). Среди других параметров СОКТ диагностической значимостью во всех клинических группах обладали показатели толщины СНВС в носовом, верхнем и нижнем квадрантах на уровне $\mathrm{p}<0,003,0,002$ и 0,0003 соответственно.

Отмечен высокий уровень статистической значимости различий в группе АЗН вследствие РС по сравнению с группами ОН воспалительной и демиелинизирующей этиологии, по кластеру (параметру) RNFL Thickness и RNFL-N (рис. 1, 2).

При рассмотрении показателя средней толщины комплекса ГКС + ВПС, полученного по данным СОКТ, выявлен высокий уровень статистической значимости различий между группами: показатели составили в среднем 80,73 мкм для ОН воспалительной этиологии, 62,88 мкм для АЗН вследствие РС и 77,34 мкм для ОН вследствие РС. Выявлен высокий уровень статистической значимости различий между группами ОН (как воспалительной, так и демиелинизирующей этиологии) и группой АЗН вследствие РС $(\mathrm{p}<0,0005)$. При этом уровень значимости различий между группами ОН воспалительной и демиелинизирующей этиологии по параметру ГКС + ВПС оказался низким. В целом на пораженном глазу данный показатель оказался ниже в каждой из клинических групп ( $\mathrm{p}=0,004)$. Наиболее высокий уровень статистической значимости различий по ГКС + ВПС выявлен для группы АЗН вследствие РС: различие значений между пораженным и парным глазами составило 58,38 и 67,38 мкм соответственно. Различие значений этих параметров пораженного и парного глаза составило 80 мКм к 81,46 мкм для ОН воспалительной этиологии и 76,50 мкм к 78,18 мкм для ОН вследствие РС. Уровень статистической значимости различий взаимодействия факторов группы и глаза был на уровне тенденции $(\mathrm{p}=0,067)$, что представлено в таблице 5 .

Таблица 4. Толщина СНВС в трех группах пациентов и их нормальные значения по протоколу СОКТ исследования ДЗН (М $\pm \delta$ ) Table 4. Retinal nerve fiber layer (RNFL) thickness in three groups of patients and its normal values according optic disc OCT protocol (M $\pm \delta$ )

\begin{tabular}{|c|c|c|c|c|}
\hline \multirow[t]{2}{*}{$\begin{array}{l}\text { Толщина СНBC } \\
\text { RNFL thickness }\end{array}$} & \multicolumn{3}{|c|}{$\begin{array}{l}\text { Клинические группы пациентов } \\
\text { Clinical groups of patients }\end{array}$} & \multirow[t]{2}{*}{$\begin{array}{l}\text { Норма } \\
\text { Normal values }\end{array}$} \\
\hline & $\begin{array}{c}\text { ОН вследствие РC } \\
\text { ON due to MS } \\
n=43\end{array}$ & $\begin{array}{c}\text { ОН воспалительной } \\
\text { этиологии } \\
\text { ON of inflammatory etiology } \\
n=13\end{array}$ & $\begin{array}{c}\text { АЗН вследствие РС } \\
\text { ONA due to MS } \\
n=62\end{array}$ & \\
\hline $\begin{array}{l}\text { CHBC-T, мкм } \\
\text { RNFL-T, } \mu \mathrm{m}\end{array}$ & $65,35 \pm 18,88$ & $74,38 \pm 24,6$ & $47,92 \pm 13,8$ & $67,78 \pm 10,12$ \\
\hline $\begin{array}{l}\text { RNFL-T парного глаза, мкм } \\
\text { RNFL-T, fellow еуе, } \mu \mathrm{m}\end{array}$ & $61,53 \pm 11,28$ & $63,46 \pm 11,77$ & $54,70 \pm 17,7$ & $67,78 \pm 10,12$ \\
\hline $\begin{array}{l}\text { RNFL-N, мкм } \\
\text { RNFL-N, } \mu \mathrm{m}\end{array}$ & $77,85 \pm 21,69$ & $95,54 \pm 61,26$ & $64,06 \pm 11,50$ & $71,71 \pm 8,54$ \\
\hline $\begin{array}{l}\text { RNFL-N парного глаза, мкм } \\
\text { RNFL-N, fellow еуе, } \mu \mathrm{m}\end{array}$ & $68,55 \pm 11,10$ & $68,85 \pm 14,36$ & $64,38 \pm 15,20$ & $71,71 \pm 8,54$ \\
\hline $\begin{array}{l}\text { RNFL-S, мКм } \\
\text { RNFL-S, } \mu \mathrm{m}\end{array}$ & $133,38 \pm 40,54$ & $155,77 \pm 77,85$ & $101,30 \pm 28,40$ & $123,07 \pm 11,24$ \\
\hline $\begin{array}{l}\text { RNFL-S парного глаза, мкм } \\
\text { RNFL-S, fellow еуе, } \mu \mathrm{m}\end{array}$ & $110,84 \pm 17,23$ & $127,92 \pm 11,36$ & $103,98 \pm 21,70$ & $123,07 \pm 11,24$ \\
\hline $\begin{array}{l}\text { RNFL-I, мкм } \\
\text { RNFL-I, } \mu \mathrm{m}\end{array}$ & $133,74 \pm 43,05$ & $161,85 \pm 97,47$ & $104,04 \pm 24,50$ & $129,92 \pm 12,14$ \\
\hline $\begin{array}{l}\text { RNFL-I парного глаза, мкм } \\
\text { RNFL-I, fellow еуе, } \mu \mathrm{m}\end{array}$ & $116,82 \pm 21,42$ & $128,31 \pm 15,27$ & $105,80 \pm 23,20$ & $129,92 \pm 12,14$ \\
\hline $\begin{array}{l}\text { RNFL, MKM } \\
\text { RNFL, } \mu \mathrm{m}\end{array}$ & $105,81 \pm 33,42$ & $121,38 \pm 63,91$ & $78,63 \pm 12,45$ & $98,21 \pm 6,76$ \\
\hline $\begin{array}{l}\text { RNFL парного глаза, мкм } \\
\text { RNFL, fellow еуе, } \mu \mathrm{m}\end{array}$ & $88,58 \pm 12,11$ & $96,92 \pm 10,11$ & $83,33 \pm 15,74$ & $98,21 \pm 6,76$ \\
\hline
\end{tabular}

Note. MS - multiple sclerosis, ON - optical neuritis, ONA - optic nerve atrophy, $\mathrm{T}$ - temporal, $\mathrm{N}-$ nasal, $\mathrm{S}-$ superior, I - inferior. 


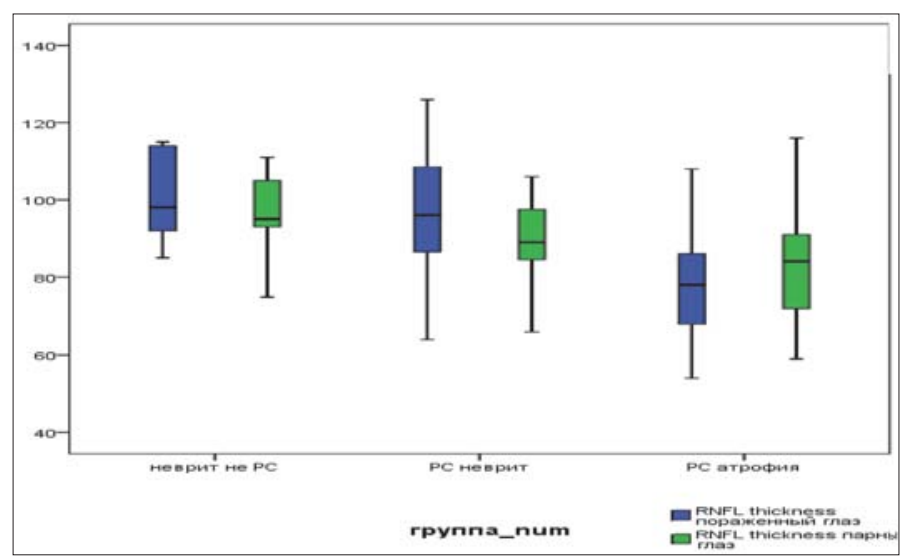

Рис. 1. Межгрупповые различия по критерию RNFL Thickness Fig. 1. Intergroup differences in the RNFL Thickness parameter

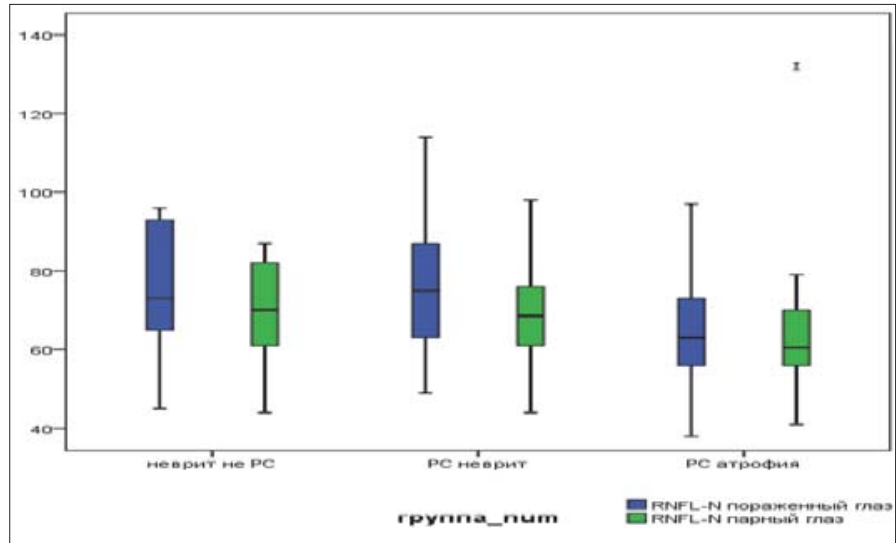

Рис. 2. Межгрупповые различия по критерию RNFL-N

Fig 2. Intergroup differences in the RNFL-N parameter

Таблица 5. Параметры ГКС в трех группах пациентов и их нормальные значения $(\mathrm{M} \pm \delta)$

Table 5. Ganglion cell layer parameters in three groups of patients and their normal values $(\bar{M} \pm \delta)$

\begin{tabular}{|c|c|c|c|c|}
\hline \multirow{2}{*}{$\begin{array}{l}\text { Параметры ОКТ: ГКС + ВПС } \\
\text { и их сегменты } \\
\text { OCT parameters: GCL + IPL protocol }\end{array}$} & \multicolumn{3}{|c|}{$\begin{array}{c}\text { Клинические группы пациентов } \\
\text { Clinical groups of patients }\end{array}$} & \multirow[t]{2}{*}{$\begin{array}{l}\text { Hopмa } \\
\text { Normal values }\end{array}$} \\
\hline & $\begin{array}{c}\text { ОН вследствие РC } \\
\text { ON due to MS } \\
n=23\end{array}$ & $\begin{array}{c}\text { ОН воспалительной } \\
\text { этиологии } \\
\text { ON of inflammatory etiology } \\
\mathrm{n}=11\end{array}$ & $\begin{array}{l}\text { АЗН вследствие } \\
\text { PC } \\
\text { ONA due to MS } \\
n=8\end{array}$ & \\
\hline $\begin{array}{l}\Gamma K C+\text { BПC, мКм } \\
\text { GCL + IPL, } \mu \mathrm{m}\end{array}$ & $76,7 \pm 5,41$ & $80,00 \pm 6,43$ & $58,37 \pm 5,63$ & $84,21 \pm 2,54$ \\
\hline $\begin{array}{l}\text { ГКС + ВПС парного глаза, мкм } \\
\text { GCL+ IPL, fellow eye, } \mu \mathrm{m}\end{array}$ & $78,18 \pm 6,62$ & $81,45 \pm 6,27$ & $67,67 \pm 11,63$ & $84,21 \pm 2,54$ \\
\hline $\begin{array}{l}\text { ГKC, верхний сегмент, мкм } \\
\text { GCL-S, } \mu \mathrm{m}\end{array}$ & $67,72 \pm 8,44$ & $63,36 \pm 8,31$ & $73,27 \pm 4,54$ & $85,07 \pm 3,62$ \\
\hline $\begin{array}{l}\text { ГKC, верхний сегмент парного глаза, мкм } \\
\text { GCL-S, fellow eye, } \mu \mathrm{m}\end{array}$ & $71,72 \pm 11,27$ & $68,57 \pm 11,61$ & $75,73 \pm 9,89$ & $85,07 \pm 3,62$ \\
\hline $\begin{array}{l}\text { ГKC, верхневисочный сегмент, мкм } \\
\text { GCL-ST, } \mu \mathrm{m}\end{array}$ & $72,36 \pm 4,15$ & $84,14 \pm 5,63$ & $63,43 \pm 8,64$ & $84,28 \pm 3,47$ \\
\hline $\begin{array}{l}\text { ГКС, верхневисочный сегмент парного } \\
\text { глаза, мKм } \\
\text { GCL-SN, fellow eye, } \mu \mathrm{m}\end{array}$ & $73,82 \pm 7,31$ & $85,50 \pm 6,17$ & $70,00 \pm 13,62$ & $84,28 \pm 3,47$ \\
\hline $\begin{array}{l}\text { ГКС нижневисочный сегмент, мкм } \\
\text { GCL-IT, } \mu \mathrm{m}\end{array}$ & $71,73 \pm 5,24$ & $83,11 \pm 5,15$ & $65,57 \pm 9,32$ & $83,57 \pm 4,50$ \\
\hline $\begin{array}{l}\text { ГКС нижневисочный сегмент парного } \\
\text { глаза, мкм } \\
\text { GCL-IT, fellow eye, } \mu \mathrm{m}\end{array}$ & $75,36 \pm 8,35$ & $84,43 \pm 5,09$ & $70,50 \pm 15,52$ & $83,57 \pm 4,50$ \\
\hline $\begin{array}{l}\text { ГКС нижний сегмент, мкм } \\
\text { GCL-I, } \mu \mathrm{m}\end{array}$ & $69,73 \pm 6,36$ & $82,13 \pm 5,80$ & $63,50 \pm 10,18$ & $81,57 \pm 4,50$ \\
\hline $\begin{array}{l}\text { ГKC, нижний сегмент парного глаза, мкм } \\
\text { GCL-I, fellow eye, } \mu \mathrm{m}\end{array}$ & $72,14 \pm 5,41$ & $82,7 \pm 4,4$ & $68,9 \pm 9,7$ & $81,57 \pm 4,50$ \\
\hline $\begin{array}{l}\text { ГКС нижневисочный сегмент, мкм } \\
\text { GCL-IT, } \mu \mathrm{m}\end{array}$ & $65,40 \pm 9,95$ & $62,36 \pm 8,31$ & $69,27 \pm 7,03$ & $84,64 \pm 3,75$ \\
\hline $\begin{array}{l}\text { ГКС, нижневисочный сегмент парного } \\
\text { глаза, мкм } \\
\text { GCL-IT, fellow eye, } \mu \mathrm{m}\end{array}$ & $72,20 \pm 9,33$ & $70,86 \pm 9,23$ & $73,91 \pm 9,61$ & $84,64 \pm 3,75$ \\
\hline $\begin{array}{l}\text { ГКС, нижненосовой сегмент, мкм } \\
\text { GCL-IN, } \mu \mathrm{m}\end{array}$ & $66,4 \pm 9,84$ & $62,64 \pm 10,88$ & $71,18 \pm 5,81$ & $85,85 \pm 3,23$ \\
\hline $\begin{array}{l}\text { ГКС нижненосовой сегмент парного глаза, } \\
\text { мKм } \\
\text { GCL-IN, fellow eye, } \mu \mathrm{m}\end{array}$ & $72,76 \pm 9,50$ & $71,43 \pm 8,94$ & $74,45 \pm 10,34$ & $85,85 \pm 3,23$ \\
\hline
\end{tabular}

Note. GCL - ganglion cells layer, IPL - intern plexiform layer, MS - multiple sclerosis, ON - optical neuritis, ONA - optic nerve atrophy, T temporal, $\mathrm{N}$ - nasal, $\mathrm{S}$ - superior, I - inferior.

По результатам теста ANOVA выявлен высокий уровень статистической значимости различий парных глаз по показателю ЭЛ. Если у пациента с ОН на парном глазу показатель ЭЛ составляет 36 Гц и более, то с чувствительностью $92 \%$ и специфичностью 48 \% можно утверждать, что это ОН недемиелинизирующей этиологии $(\mathrm{p}=0,019)$.

Регрессионный анализ. Выявлено, что параметры микропериметрии обладают низкой чувствительностью и 
специфичностью в дифференциальной диагностике ОН и АЗН вследствие РС, поэтому различить ОН и АЗН при РС методом микропериметрии невозможно. Однако при сравнении показателей микропериметрии в группах ОН и АЗН с нормативными значениями, полученными при обследовании здоровых испытуемых, были выявлены достоверные различия $(\mathrm{p}<0,05)$ по параметру средней светочувствительности сетчатки в пределах до $6^{\circ}$ от центра поля зрения (табл. 6).

При оценке диагностической значимости СОКТ толщины перипапиллярного CHBC/RNFL Thickness получены следующие показатели ROC-кривой: $\mathrm{AUC}=82,7$ \%, ОШ $=0,043$ ( $<<0,0001)$. Так, если у пациента с РС толщина перипапиллярного СНВС составляет 62 мкм и менее, то с чувствительностью $100 \%$ и специфичностью $93 \%$ это свидетельствует об АЗН на данном глазу.

Для параметра комплекса ГКС + ВПС / GCL + IPL $\mathrm{AUC}=98,6 \%$, ОШ = 0,017 (p < 0,0001); для параметра ГКС + ВПС в нижнем сегменте / GCL-I AUC $=72,1 \%$, ОШ =0,107 $(\mathrm{p}=0,063)$; для параметра ГКС + ВПС в верхнем сегменте / GCL-S AUC = 83,8 \%, ОШ = 0,082 (p=0,004). Так, если у пациента с РС показатель ГКС + ВПС составляет 52 мкм и менее, то с чувствительностью $100 \%$ и специфичностью 87,5 \% это свидетельствует о наличии на данном глазу АЗН вследствие РС. Признаками АЗН вследствие РС у пациента также является снижение показателя ГКС + ВПС в нижнем сегменте: если он составляет 50 мкм и менее (чувствительность $100 \%$ и специфичность $92 \%$ ) - и/или ГКС + ВПС в верхнем сегменте составляет 51 мкм и менее (чувствительность $100 \%$ и специфичность $93 \%$ ).

Таким образом, выявлены критерии диагностики АЗН у пациентов с РС, обследованных впервые, если у них диагноз «атрофия зрительного нерва» был установлен на основании снижения остроты зрения, истончения СНВС по данным СОКТ и данным офтальмоскопии и ранее не был документирован.

\section{ОБСУЖДЕНИЕ}

Нами сопоставлены и обработаны методами статистики высокого порядка данные электрофизиологического, структурного и функционального анализа зрительных функций трех групп пациентов: с ОН при манифестации РС, с АЗН вследствие РС и с ОН воспалительной этиологии. Детальное описание офтальмологического статуса и результатов обследования пациентов для каждой группы в отдельности было представлено в наших предыдущих исследованиях [12-15]. Методом корреляционного анализа выявлено, что на пораженном (худшем в функциональном отношении) глазу параметры электрофизиологического исследования (ПЭЧ и ЭЛ) имеют сильную двустороннюю корреляционную связь с показателем средней светочувствительности сетчат- ки в пределах $6^{\circ}$ от центра поля зрения, подтвержденную как минимум тремя статистическими тестами. Эти данные отражают преобладание функциональных нарушений на пораженном глазу при РС. Кроме того, ПСС сетчатки имеет корреляционные связи с параметрами СОКТ - СНВС, толщиной верхневисочного и верхнего секторов ГКС + ВПС, что может быть расценено как тенденция к ранним структурным нарушениям в проекции зрительного анализатора (3А) при РС. Данные литературы свидетельствуют о первостепенном значении изучения комплекса ГКС + ВПС в оценке процесса глобальной нейроаксональной дегенерации при РС [15-19]. В нашем исследовании пациенты с РС не имели ранее документированный другими методами исследования диагноз АЗН, поэтому мы считаем, что показатель ГКС + ВПС является определяющим в подтверждении диагноза АЗН вследствие РС, так как снижение показателей данного комплекса наблюдается раньше, чем снижение толщины СНВС и других структурно-функциональных показателей.

По результатам многомерного анализа всех клинических групп пациентов выявлены и подтверждены значимые при поражении ЗА вследствие РС диагностические параметры - снижение ПСС сетчатки в пределах до $6^{\circ}$ от центра поля зрения по данным микропериметрии ( $<<0,0005)$, уменьшение толщины СНВС $(\mathrm{p}<0,005)$ и комплекса ГКС + ВПС по данным СОКТ $(\mathrm{p}=0,004)$. При проведении внутригрупповых сравнений выявлена диагностическая значимость СОКТ в ранней диагностике АЗН вследствие РС ( $<$ <0,0005). Рядом исследователей предложено использовать параметр средней толщины СНВС по данным СОКТ как диагностический критерий прогрессирования и оценки тяжести течения РС [20-23], однако это предложение на сегодняшний день не получило широкого распространения. Тем не менее на основании полученных нами результатов применения методов статистической обработки большой выборки данных мы считаем, что критерий толщины СНВС может быть отнесен к показателям тяжести течения заболевания международной шкалы EDSS.

Выявлен высокий уровень статистической значимости различий между нормальными значениями ПСС сетчатки в пределах до $6^{\circ}$ от центра поля зрения и значениями, полученными у пациентов с ОН и АЗН вследствие РС. Метод микропериметрии позволяет выявить центральные и/или парацентральные дефекты в поле зрения, определить снижение средней светочувствительности в центральной зоне сетчатки и изменение стабильности фиксации у пациентов с ОН и АЗН вследствие РС. Преимущество микропериметрии перед компьютерной периметрией в диагностике центральных дефектов поля зрения при ОН подтверждено рядом наших работ и изобретений [24-26]. В прежних исследованиях нами было показано, что снижение ПСС с вы-

Таблица 6. Параметры микропериметрии в трех группах пациентов и их нормальные значения $(\mathrm{M} \pm \delta)$

Table 6. Parameters of microperimetry in three groups of patients and their normal values $(\mathrm{M} \pm \delta)$

\begin{tabular}{|c|c|c|c|c|}
\hline \multirow[t]{2}{*}{$\begin{array}{l}\text { Параметры микропериметрии } \\
\text { Parameters of microperimetry }\end{array}$} & \multicolumn{3}{|c|}{$\begin{array}{l}\text { Клинические группы } \\
\text { Clinical groups }\end{array}$} & \multirow[t]{2}{*}{$\begin{array}{c}\text { Норма } \\
\text { Normal values }\end{array}$} \\
\hline & $\begin{array}{c}\text { ОН вследствие РС } \\
\text { ON due to MS } \\
n=28\end{array}$ & $\begin{array}{c}\text { ОН воспалительной } \\
\text { этиологии } \\
\text { ON of inflammatory etiology } \\
n=12\end{array}$ & $\begin{array}{c}\text { АЗН вследствие РС } \\
\text { ONA due to MS } \\
n=8\end{array}$ & \\
\hline $\begin{array}{l}\text { ПСС } 6^{\circ}, \text { дБ } \\
\text { Mean sensitivity } 6^{\circ}, \mathrm{dB}\end{array}$ & $10,87 \pm 6,07^{*}$ & $10,87 \pm 6,07^{*}$ & $12,58 \pm 6,60^{*}$ & $18,83 \pm 4,10^{*}$ \\
\hline $\begin{array}{l}\text { ПСС 6º } 6^{\circ} \text { парный глаз, дБ } \\
\text { Mean sensitivity } 6^{\circ} \text {, fellow eye, dB }\end{array}$ & $17,68 \pm 2,24$ & $17,68 \pm 2,24$ & $18,68 \pm 0,74$ & $18,83 \pm 4,10$ \\
\hline
\end{tabular}

Примечание. ${ }^{*} \mathrm{p}<0,05$.

Note. ${ }^{*} \mathrm{p}<0.05$, MS - multiple sclerosis, ON - optical neuritis, ONA - partial optic nerve atrophy. 
явлением центральных дефектов в поле зрения по данным микропериметрии является патогномоничным признаком ОН вследствие манифестации РС [14, 25]. Тем не менее для подтверждения диагноза АЗН вследствие РС необходимо дополнительное применение метода СОКТ.

Методом бинарной логистической регрессии выявлен высокий уровень статистической значимости различий между группами ОН и АЗН вследствие РС на пораженном (или худшем в функциональном отношении) глазу по показателю толщины СНВС $(\mathrm{p}=0,008)$. Кроме того, высокий уровень значимости различий получен по кластеру (параметру) верхнего височного сегмента комплекса ГКС + ВПС на уровне $\mathrm{p}=0,033$ и параметру нижнего височного сегмента комплекса ГКС + ВПС $(\mathrm{p}=0,05)$. Отмечен высокий уровень статистической значимости различий указанных показателей при АЗН вследствие прогрессирования РС.

Таким образом, обследование пациента со зрительными нарушениями вследствие РС с помощью методов структурно-функционального анализа позволяет с высокой достоверностью диагностировать ОН или АЗН демиелинизирующей этиологии, провести дифференциальную диагностику с ОН воспалительной этиологии.

\section{ЗАКЛЮЧЕНИЕ}

Данные корреляционного анализа имеют клиническую значимость и демонстрируют тенденцию к ранним структурным нарушениям в проекции зрительного анализатора при РС, так как потеря объема комплекса ГКС + ВПС отражает процесс прогрессирования РС в целом, что согласуется с мнением преобладающего большинства исследователей как офтальмологического, так и неврологического профиля. Результаты, полученные при оценке диагностической значимости СОКТ и микропериметрии методами многомерного и регрессионного анализа, выявляют критерии развития патологических процессов, связанных с прогрессированием РС: среднюю светочувствительность сетчатки в пределах до $6^{\circ}$ от центра поля зрения, толщину СНВС, ГКС + ВПС, в том числе в верхнем, верхневисочном и нижнем сегментах. Полученные различия являются значимыми в дифференциальной диагностике ОН воспалительной и демиелинизирующей этиологии, а также верификации прогрессирования АЗН вследствие РС.

\section{Лumepamypa/References}

1. Давыдовская М.В., Цысарь М.А., Бойко А.Н. идр. Повреждение комплекса ганглиозных клеток и слоя нервных волокон сетчатки при рассеянном склерозе. Журнал неврологии и психиатрии им. С.С. Корсакова. Спецвыпуски. 2012; 2 (2): 47-51. [Davydovskaya M.V., Tsysar M.A., Boyko A.N., et al. Damage of a complex of ganglion cells and a layer of retinal nerve fibers in multiple sclerosis. S.S. Korsakov Journal of neurology and psychiatry. 2012; 2 (2): 47-51 (in Russian)].

2. Гусев Е.И., Завалишин И.А., Бойко А.Н. Рассеянный склероз: клиническое руководство, Москва; 2011. [Gusev E.I., Zavalishin I.A., Boiko A.N. Multiple sclerosis, clinical guidelines, Moscow; 2011 (in Russian)].

3. Balcer L.J., Miller D.H., Reingold S.C., Cohen J.A. Vision and vision-related outcome measures in multiple sclerosis. Brain. 2015; 138 (1): 11-27. doi: 10.1093/brain/awu335

4. Мороз Н.Г. Характеристика лиц молодого возраста, повторно признанных инвалидами вследствие болезней нервной системы, в Российской Федерации в 2001-2006 гг. Медико-социальная экспертиза и реабилитация. 2008; 2: 27-9. [Moroz N.G. Characteristics of young subjects reconfirmed as disabled due to diseases of the nervous system in the Russian Federation in 2001-2006. Medical and social examination and rehabilitation. 2008; 2: 27-9 (in Russian)].

5. Нероев В.В., Зуева М.В., Цапенко И. В. идр. Нейродегенеративные изменения в сетчатке у больных ремитирующим рассеянным склерозом и ретробульбарным невритом: морфофункциональные параллели. Российский офтальмологический журнал. 2012; 5 (4): 63-8. [Neroev V.V., Zueva M.V., Tsapenko I.V., et al. Neurodegenerative changes in the retina in patients with remitting multiple sclerosis and retrobulbar neuritis: morphofunctional parallels.
Russian ophthalmological journal. 2012; 5 (4): 63-8 (in Russian)].

6. Нероев В.В., Елисеева Е.К., Зуева М.В. идр. Демиелинизирующий оптический неврит: корреляция данных оптической когерентной томографии и мультифокальной электроретинографии. Анналы клинической и экспериментальной неврологии. 2014; 8 (2): 22-6. [Neroev V.V., Eliseeva E.K., Zueva M.V., et al. Demyelinating optical neuritis: correlation of optical coherence tomography and multifocal electroretinography. Annals of clinical and experimental neurology. 2014; 8 (2): 22-6 (in Russian)]

7. Шnак А.А. Вопросы статистического анализа в российских офтальмологических журналах. Офтальмохирургия. 2016; (1): 73-7. https://doi. org/10.25276/0235-4160-2016-1-73-77 [Shpak A.A. Issues of the statistical analysis in the Russian ophthalmic journals. Fyodorov Journal of Ophthalmic Surgery. 2016; (1): 73-7 (in Russian). https://doi.org/10.25276/0235-41602016-1-73-77]

8. Olsen C.H. Statistics in Infection and immunity revisited. Infect. Immun. 2014; 82 (3): 916-20. doi: 10.1128/IAI.00811-13

9. Lisboa R., Meira-Freitas D., Tatham A.J., et al. Use of statistical analyses in the ophthalmic literature. Ophthalmology. 2014; 121 (7): 1317-21. doi:10.1016/j. ophtha.2014.01.015

10. Schulz C.B., Kennedy A., Rymer B.C. Trends in ophthalmology journals: a five-year bibliometric analysis (2009-2013). Int. J. Ophthalmol. 2016; 9 (11): 1669-75. doi:10.18240/ijo.2016.11.22

11. Ying G.S., Maguire M.G., Glynn R., Rosner B. Tutorial on Biostatistics: Linear regression analysis of continuous correlated eye data. Ophthalmic Epidemiol. 2017; 24 (2): 130-40. doi:10.1080/09286586.2016.1259636

12. Иойлева Е.Э., Кривошеева М.С., Смирнова М.А. Односторонний отек зрительного нерва: особенности дифференциальной диагностики. Таврический медико-биологический вестник. 2013; 3 (2): 166-70. [Ioyleva E.E., Krivosheva M.S., Smirnova M.A. Unilateral swelling of the optic nerve: features of differential diagnosis. Tavricheskij mediko-biologicheskij vestnik. 2013; 3 (2): 166-70 (in Russian)].

13. Иойлева Е.Э., Кривошеева М.С., Макаренко И.Р. Исследование асимметрии параметров диска зрительного нерва при рассеянном склерозе по данным ОКТ. Вестник Тамбовского университета. Серия: Естественные и технические науки. 2016; 21 (4): 1559-63. doi: 10.20310/1810-01982016-21-4-1559-1563 [Ioyleva E.E., Krivosheva M.S., Makarenko I.R. Study of asymmetric parameters of the optic disk nerve for multiple sclerosis in OCT. Bulletin of Tambov University. Series: Natural and technical sciences. 2016; 21 (4): 1559-63 (in Russian). doi: 10.20310/1810-0198-2016-21-4-1559-1563]

14. Иойлева Е.Э., Кривошеева М.С. Дифференциальная диагностика оптического неврита различной этиологии методами функциональной диагностики. Инфекция, иммунитет и фармакология. 2016; 5: 57-60. [Ioyleva E.E., Krivosheeva M.S. Differential diagnosis of optical neuritis of various etiologies by functional diagnostic methods. Infection, immunity and pharmacology. 2016; 5: 57-60 (in Russian)].

15. Иойлева Е.Э., Кривошеева М.С. Микропериметрия при оптическом неврите вследствие рассеянного склероза. Офтальмохирургия. 2016; 3: 33-8/ https://doi.org/10.25276/0235-4160-2016-3-33-38 [Ioyleva E.E., Krivosheeva M.S. Microperimetry in optic neuritis due to multiple sclerosis. Fyodorov journal of ophthalmic surgery. 2016; (3): 33-8 (in Russian). https:// doi.org/10.25276/0235-4160-2016-3-33-38]

16. Камилов Х.М., Касымова М.С., Хамраева Г.Х. Оценка эффективности лечения оптического неврита методом магнитно-резонансной томографии. Офтальмохирургия. 2015; 1: 38-42. [Kamilov H.M., Kasymova M.S., Khamrayeva G.Kh. Evaluation of the effectiveness of treatment of optical neuritis by magnetic resonance imaging. Fyodorov journal of ophthalmic surgery. 2015; 1: 38-42 (in Russian)].

17. Нероев В.В., Зуева М.В., Лысенко В.С. и др. Оптический неврит. В кн. Завалишин И.А., Пирадов М.А., Бойко А.Н., ред. Аутоиммунные заболевания в неврологии. Клиническое руководство. Москва; 2014: 66-102. [Neroev V.V., Zueva M.V., Lysenko V.S., et al. Optical neuritis. In: Zavalishin I.A., Piradov M.A., Boyko A.N., eds. Autoimmune diseases in neurology. Clinical Guidelines. Moscow; 2014: 66-102 (in Russian)].

18. Рябцева А.А., Андрюхина О.М., Котов С.В., Якушина Т.И., Кучина Н.В. Морфометрические характеристики сетчатки и зрительного нерва у пациентов с рассеянным склерозом разных возрастных групп. Клиническая геронтология. 2014; 20 (7-8): 21-4. [Ryabtseva A.A., Andryukhina O.M., Kotov S.V., Yakushina T.I., Kuchina N.V. Morphometric characteristics of the retina and optic nerve in patients with multiple sclerosis of different age groups. Clinical gerontology. 2014; 20 (7-8): 21-4 (in Russian)].

19. Андрюхина О.М., Рябцева А.А., Котов С.В., Якушина Т.И., Кучина Н.В. Мониторинг офтальмологических индикаторов у больных рассеянным склерозом. Альманах клинической медицины. 2015; 36: 53-8. https://doi. org/10.18786/2072-0505-2015-36-53-58 [Andryukhina O.M., Ryabtseva A.A., Kotov S.V., Yakushina T.I., Kuchina N.V. Monitoring of ophthalmological indicators in patients with multiple sclerosis. Almanac of clinical medicine. 2015; 36: 53-8 (in Russian). https://doi.org/10.18786/2072-0505-2015-36-53-58]

20. Маслова Н.Н., Андреева Е.А. Возможности нейроофтальмологического 
обследования в ранней диагностике рассеянного склероза. Медицинский альманах. 2013; 29 (5): 201-3. [Maslova N.N., Andreeva E.A. Possibilities of neurological examination in the early diagnosis of multiple sclerosis. Meditsinskiy almanakh, 2013; 29 (5): 201-3 (in Russian)].

21. Акопян В.С., Бойко А.Н., Давыдовская М.В. и др. Нейроархитектоника сетчатки при рассеянном склерозе: диагностические возможности оптической когерентной томографии (предварительные результаты). Офтальмология. 2011; 1: 32-6. [Akopyan V.S., Boyko A.N., Davydovskaya M.V., et al. Neuroarchitecture of the retina in multiple sclerosis: diagnostic capabilities of optical coherence tomography (preliminary results). Ophthalmology. 2011; (1): 32-6 (in Russian)].

22. Кучина Н.В., Якушина Т.И., Котов С.В. и др. Исследование цветового зрения для диагностики и динамического наблюдения при рассеянном склерозе. Альманах клинической медицины. 2015; 36: 47-52. https:// doi.org/10.18786/2072-0505-2015-36-47-52 [Kuchina N.V., Yakushina T.I., Kotov S.V., et al. The study of color vision for diagnosis and dynamic observation in multiple sclerosis. Almanac of clinical medicine. 2015; 36: 47-52 (in Russian). https://doi.org/10.18786/2072-0505-2015-36-47-52]
23. Garcia-Martin E., Rodriguez-Mena D., Herrero R., et al. Neuro-ophthalmologic evaluation, quality of life, and functional disability in patients with MS. Neurology. 2013; (81): 76-83. doi: 10.1212/WNL.0b013e318299ccd

24. Иойлева Е.Э., Гаджиева Н.С., Кривошеева М.С., Кутейникова М.Э. Способ ранней диагностики ретробульбарного неврита при дебюте рассеянного склероза. Патент РФ № 2548511 от 29.11.2013. [Ioyleva E.E., Gadzhiyeva N.S., Krivosheeva M.S., Kuteynikova M.E. A method for early diagnosis of retrobulbar neuritis at the debut of multiple sclerosis. Patent RU No. 2548511, priority of 11.29.2013 (in Russian)].

25. Иойлева Е.Э., Кривошеева М.С. Способ диагностики монокулярного оптического неврита в дебюте рассеянного склероза. Патент РФ № 2562136 от 13.11.2014. [Ioyleva E.E., Krivosheeva M.S. A method for diagnosing monocular optic neuritis in the debut of multiple sclerosis. Patent RU No. 2562136, priority of 13.11.2014 (in Russian)].

26. Ioyleva E., Krivosheeva M. Microperimetry in the diagnosis of the first manifestation of optic neuritis in multiple sclerosis. Journal of the Neurological Sciences. 2015; 357 (1), e 47. https://doi.org/10.1016/j.jns.2015.08.196

Вклад авторов в работу: М.С. Кривошеева - обработка материала, написание и научное редактирование текста; Е.Э. Иойлева - дизайн исследования и научное консультирование.

Поступила: 05.07.2019

Переработана: 26.09.2019

Принята к печати: 01.11.2019
Originally received: 05.07.2019

Final revision: 26.09 .2019

Accepted: 01.11.2019

\section{ИНФОРМАЦИЯ ОБ АВТОРАХ/INFORMАТION AВOUT THE AUTHORS}

ГБУЗ Московской области «Сергиево-Посадская районная больница», Новоугличское шоссе, д. 62а, Московская область, г. Сергиев Посад, 141301, Россия

Мария Сергеевна Кривошеева - канд. мед. наук, врач-офтальмолог хирургического отделения поликлиники

${ }^{\prime}$ ФГУУ НМИЦ «Межотраслевой научно-технический комплекс "Микрохирургия глаза" им. акад. С.Н. Федорова» Минздрава России, Бескудниковский бульвар, д. 59а, Москва, 127486, Россия

${ }^{2}$ МГмСУ им. А.С. Евдокимова, ул. Делегатская, д. 20, стр. 1, Москва, 127473, Россия

Елена Эдуардовна Иойлева - д-р мед. наук, ученый секретарь ${ }^{1}$, профессор кафедры глазных болезней ${ }^{2}$

Для контактов: Мария Сергеевна Кривошеева, krivosheeva_ms@mail.ru
City hospital of Sergiev Posad RB, Novouglichskoe highway, 62a, Sergiev Posad, Moscow region, 141301, Russia

Maria S. Krivosheeva - Cand. of Med. Sci., ophthalmologist, surgical department of the polyclinic

${ }^{1}$ S.N. Fyodorov Eye Microsurgery NMRC, 59a, Beskudnikovsky Bulvar, Moscow, 127486, Russia

${ }^{2}$ A.I. Evdokimov Moscow State University of Medicine and Dentistry, 20, p. 1, Delegatskaja St., Moscow, 127473, Russia

Elena E. Ioyleva - Dr. of Med. Sci., scientific secretary ${ }^{1}$, professor, chair of ophthalmology ${ }^{2}$

Contact information: Maria S. Krivosheeva, krivosheeva_ms@mail.ru 